\title{
Phosphoproteomic analysis of mammalian infective Trypanosoma brucei subjected to heat shock suggests atypical mechanisms for thermotolerance
}

\author{
Cher P. Ooi ${ }^{\mathrm{a}, *}$, Corinna Benz ${ }^{\mathrm{b}, 1}$, Michael D. Urbaniak ${ }^{\mathrm{b}, *}$ \\ ${ }^{a}$ Department of Life Sciences, Sir Alexander Fleming Building, Imperial College London, South Kensington, London SW7 2AZ, UK \\ ${ }^{\mathrm{b}}$ Biomedical and Life Sciences, Faculty of Health and Medicine, Lancaster University, Lancaster LA1 4YG, UK
}

\section{A R T I C L E I N F O}

\section{Keywords:}

African sleeping sickness

Trypanosoma brucei

heat shock

SILAC

phosphoproteomics

\begin{abstract}
A B S T R A C T
The symptoms of African sleeping sickness, caused by the parasite Trypanosoma brucei, can include periods of fever as high as $41^{\circ} \mathrm{C}$ which triggers a heat shock response in the parasite. To capture events involved in sensing and responding to heat shock in the mammalian infective form we have conducted a SILAC-based quantitative proteomic and phosphoproteomic analysis of $T$. brucei cells treated at $41{ }^{\circ} \mathrm{C}$ for $1 \mathrm{~h}$. Our analysis identified 193 heat shock responsive phosphorylation sites with an average of 5-fold change in abundance, but only 20 heat shock responsive proteins with average of 1.5-fold change. These data indicate that protein abundance does not rapidly respond $(\leq 1 \mathrm{~h}$ ) to heat shock, and that the changes observed in phosphorylation site abundance are larger and more widespread. The heat shock responsive phosphorylation sites showed enrichment of RNA binding proteins with putative roles in heat shock response included P-body / stress granules and the eukaryotic translation initiation 4F complex. The ZC3H11-MKT1 complex, which stabilises mRNAs of thermotolerance proteins, appears to represent a key signal integration node in the heat shock response.

Significance: We report the first quantitative study of changes in protein and phosphorylation site abundance in response to heat shock in the clinically relevant form of the human parasite Trypanosoma brucei. The identification of heat shock responsive phosphorylation sites on proteins with putative roles in thermotolerance including the ZC3H11-MKT1 complex provides evidence of the role dynamic phosphorylation of RNA binding proteins in co-ordinating heat shock. Temperature changes in the host are a major physiological challenge to parasites and factors conferring tolerance to heat shock constitute overlooked virulence factors. A better understanding of these virulence factors will pave the way for the development of novel drug therapies which selectively target $T$. brucei.
\end{abstract}

\section{Introduction}

Trypanosoma brucei are extracellular eukaryotic parasites transmitted by the bite of an infected Tsetse fly which cause African sleeping sickness in humans and the related wasting disease Nagana in livestock and wild game. There are currently 70 million people at risk across Africa, with agricultural losses projected at $\$ 2.5$ billion over the next two decades due to this disease [1,2]. African sleeping sickness is fatal if allowed to progress to a late stage of the disease and there are few treatment alternatives if resistance to front line therapeutics arise [3].

Symptoms of African sleeping sickness include cyclical fluctuations in parasite burden that coincide with periods of fever [4], which in some cases can be as high as $41^{\circ} \mathrm{C}$ [5]. Moreover, the body temperature of domestic cattle can increase up to $2^{\circ} \mathrm{C}$ from physiological norms over the course of a day [6,7], and hosts in the wild, such as African gazelles, can achieve body temperatures of up to $43^{\circ} \mathrm{C}$ during physical exertion [8]. How do these extracellular parasites adapt to periods when the temperature of the host is elevated beyond normal physiological conditions? Typically, eukaryotic cells respond to heat shock (HS) in two ways: they trigger a general arrest in protein translation to prevent further accumulation of misfolded proteins, and increase the expression of HS proteins (Hsps), which aid protein folding and degradation, despite the global block in protein translation. The eukaryotic HS response is conserved in T. brucei [9-11], but the mechanisms mediating this response appear to be divergent.

In mammalian cells, the HS translation arrest is triggered by the phosphorylation of the translation factor eIF2 $\alpha$ which blocks translation initiation. This mechanism is not conserved across all eukaryotic

\footnotetext{
* Corresponding authors.

E-mail addresses: cher.ooi@imperial.ac.uk (C.P. Ooi), m.urbaniak@lancaster.ac.uk (M.D. Urbaniak).

${ }^{1}$ Current address: Institute of Parasitology, Biology Centre, Czech Academy of Sciences, 37005 České Budějovice, Czech Republic.
} 
organisms, as the HS-triggered translation arrest in yeast and fruit flies is not via eIF2 $\alpha$ phosphorylation $[9,12,13]$. It is unknown if phosphorylation of eIF2 $\alpha$ triggers the translation arrest in T. brucei during HS as previous mutational analysis have yielded negative results [9,14]. Therefore, the translation arrest during HS in T. brucei could be triggered either by an unknown phosphorylation of eIF $2 \alpha$ or via an eIF2 $\alpha$-independent mechanism.

Up-regulation of Hsp expression is key to survival during HS, and this is regulated at the level of transcription in mammalian cells. HS increases binding of the transcription factor HSF1 to promoters upstream of $H s p$ genes, increasing the processivity of RNA polymerase II which up-regulates Hsp expression [15]. This mechanism of regulation cannot operate in $T$. brucei as genes with diverse functions are arranged in long polycistronic arrays under a common transcription promoter. Control of gene expression is primarily post-transcriptional, with RNA binding proteins (RBPs) regulating mRNA levels [16]. RBPs have been demonstrated to bind to some Hsp mRNAs in T. brucei with the likely role of stabilising them to maintain expression during HS. The zinc finger protein ZC3H11 is an RBP which has been extensively documented to stabilise HS responsive mRNAs in the insect infective (procyclic form, PCF) life cycle stage of $T$. brucei by binding to AU-rich elements $[17,18]$. ZC3H11 is only essential in the mammalian infective (bloodstream form, BSF) life cycle stage of T. brucei, yet does not stabilise HS-related mRNAs to the same degree as in PCFs [18], suggesting that other mechanisms, such as other RBPs, may instead be involved.

In this work, we provide an insight into the changes in BSF T. brucei during HS using SILAC-based quantitation of changes in protein and phosphorylation site abundance. In order to focus on signal transduction events involved in sensing and responding to heat shock, we have analysed cells after treatment at $41{ }^{\circ} \mathrm{C}$ for $1 \mathrm{~h}$. Phosphopeptides were separated from peptides using Fe-IMAC [19] and fractioned with high $\mathrm{pH}$ reverse phase spin columns [20] prior to LC-MS/MS analysis using multistage activation in the LTQ ion trap [21], allowing the quantitation and localisation of $>8000$ phosphorylation sites and $>2500$ proteins. This work, to our knowledge, constitutes the first documentation of proteomic and phosphoproteomic changes in mammalian-infective BSF T. brucei in response to HS.

\section{Material and methods}

\subsection{Trypanosome strains and culturing}

All experiments were carried out with BSF Trypanosoma brucei brucei Lister 427 strain. These cells are maintained in culture but remain infective to mice. Cells were cultured in HMI-9 medium supplemented with $15 \%$ foetal calf serum. To assess cell viability after HS, cultures at a density of $10^{5}$ cells $/ \mathrm{ml}$ were subjected to $41^{\circ} \mathrm{C}$ for different amounts of time in a water bath before being transferred back to normal growth conditions at $37^{\circ} \mathrm{C}$. Cell viability was assessed by counting only motile cells with a Neubauer haemocytometer.

\subsection{RNA manipulation and $q P C R$ analysis}

Total RNA was isolated from cells treated to HS with a Qiagen RNeasy Mini Kit (Qiagen). Genomic DNA was removed using Turbo DNase (Ambion) according to manufacturer's instructions. Synthesis of cDNA was carried out using an Omniscript RT kit (Qiagen) with $100 \mathrm{ng}$ total RNA per reaction. qPCR reactions were assembled using Brilliant II SYBR low ROX master mix (Agilent) using $1 \mu$ l of diluted cDNA (1/100, equivalent to cDNA generated from $0.5 \mathrm{ng}$ of purified total RNA). Primers used are listed in Supplementary Table S1. qPCR data are presented as fold changes using the Livak method [22]. For transcripts with $\geq 2$-fold change, a pairwise t-test was used to determine the significance of difference between the $\mathrm{Ct}$ of each time point with that at time 0 , with significance was defined as $<0.05$ of adjusted $\mathrm{P}$ value.

\subsection{Stable isotope labelling of T. brucei cells}

The stable isotope labelling by amino acids in cell culture (SILAC) labelling of BSF T. brucei cells was performed as described previously [23,24]. In brief, log phase were diluted 10,000-fold into HMI11-SILAC (HMI9 medium lacking Serum plus, L-arginine and L-lysine) supplemented with $10 \%$ dialysed FCS (1000 MWCO, Dundee Cell Products) and the standard concentration of either normal isotopic abundance $\mathrm{L}^{-}$ arginine and L-lysine (HMI11 + R0K0, referred to as Light) or ${ }^{13} \mathrm{C}_{6},{ }^{15} \mathrm{~N}_{4}$ L-arginine and ${ }^{13} \mathrm{C}_{6},{ }^{15} \mathrm{~N}_{2}$ L-lysine (HMI11 + R10K8, referred to as Heavy). The stable isotope-labelled amino acids were obtained from CK Isotopes, UK. After three days growth ( $>9$ cell divisions), the cell reached $\sim 2 \times 10^{6}$ cells $/ \mathrm{ml}$, and the Light labelled cells were heat shocked at $41{ }^{\circ} \mathrm{C}$ for $1 \mathrm{~h}$ in a water bath. Cells were harvested by centrifugation and lysed hypotonically in lysis buffer (0.1 $\mu \mathrm{M}$ 1-chloro-3tosylamido-7-amino-2-heptone (TLCK), $1 \mathrm{mM}$ benzamidine, $1 \mathrm{mM}$ phenyl-methyl sulfonyl fluoride (PMSF), $1 \mu \mathrm{g} / \mathrm{mL}$ leupeptin, $1 \mu \mathrm{g} / \mathrm{mL}$ aprotinin and Phosphatase Inhibitor Mixture II (Calbiochem)) at $1 \times 10^{9}$ cells $/ \mathrm{mL}$ for $5 \mathrm{~min}$ on ice and stored at $-80^{\circ} \mathrm{C}$ prior to subsequent processing.

\subsection{Proteomic sample preparation}

Samples were thawed and equal cell numbers $\left(2 \times 10^{8}\right.$ cells per sample) combined, then the proteins solubilized with SDS and tryptic peptides generated by an adaptation of the filter aided sample preparation procedure as described previously [24,25]. The digested peptide solution was then removed from the column, diluted to $3 \mathrm{ml}$ with $\mathrm{ABC}$ and acidified with $0.1 \%$ TFA before desalting using a $500 \mathrm{mg}$ $\mathrm{C}_{18}$ column (SepPak, Waters), lyophilisation and storage at $-80{ }^{\circ} \mathrm{C}$.

Separation of peptides from phosphopeptides using Fe-IMAC was performed as described by Ruprecht et al [19] with minor modifications as described previously [20]. Samples were subjected to HPLC (LC Packing Famos) on a Fe-IMAC column $(4 \times 50 \mathrm{~mm}$ ProPac IMAC-10, Thermo Fisher Scientific) with peptide eluted in Buffer A (30\% MeCN, $0.07 \%$ TFA) and phosphopeptide eluted in a gradient of Buffer B (30\% $\mathrm{MeCN}, 0.5 \% \mathrm{NH}_{4} \mathrm{OH}$ ) with elution monitored by absorbance at $280 \mathrm{~nm}$. Early eluting fractions $(<6 \mathrm{~min}$ ) corresponding to peptides and later eluting fractions corresponding to phosphopeptides ( $\sim 30 \mathrm{~min})$ were lyophilised and stored at $-80{ }^{\circ} \mathrm{C}$ prior to further processing.

Peptide and phosphopeptide were separately fractionated using a High $\mathrm{pH}$ Fractionation kit (ThermoFisher) according to the manufacturer's instructions with the following modifications. Lyophilised (phospho)peptides were resuspended in $100 \mu \mathrm{l} 5 \mathrm{mM} \mathrm{NH} \mathrm{N}_{4} \mathrm{OH}$ and applied to the pre-treated columns (MeCN, $0.1 \%$ TFA, and two $5 \mathrm{mM}$ $\mathrm{NH}_{4} \mathrm{OH}$ washes). The flow-through was reapplied before being collected and desalted using $\mathrm{C}_{18}$ microspin columns (Harvard Apparatus). The columns were then washed 3 times with $5 \mathrm{mM} \mathrm{NH}_{4} \mathrm{OH}$ and the (phospho)peptides eluted with different concentrations of $\mathrm{MeCN}$ (2, 3, 4, 6, 10 and $50 \% \mathrm{MeCN})$. The eluates were concatenated into five fractions as follows: $\mathrm{F} 1=2 \%+50 \%$ eluates, $\mathrm{F} 2=3 \%$ eluate, $\mathrm{F} 3=4 \%$ eluate, $\mathrm{F} 4=6 \%$ eluate, $\mathrm{F} 5=10 \%$ eluate + desalted flow through. The concatenated fractions were then lyophilised prior to analysis by LC-MS/MS.

\subsection{Mass spectrometry data acquisition}

Liquid chromatography tandem mass spectrometry (LC-MS/MS) was performed by the FingerPrints Proteomic Facility at the University of Dundee. Liquid chromatography was performed on a fully automated Ultimate U3000 Nano LC System (Dionex) fitted with a $1 \times 5 \mathrm{~mm}$ PepMap $\mathrm{C}_{18}$ trap column and a $75 \mu \mathrm{m} \times 15 \mathrm{~cm}$ reverse phase PepMap $\mathrm{C}_{18}$ nanocolumn (LC Packings, Dionex). Samples were loaded in $0.1 \%$ formic acid (buffer A) and separated using a binary gradient consisting of buffer A ( $0.1 \%$ formic acid) and buffer B ( $90 \%$ MeCN, $0.08 \%$ formic acid). Peptides were eluted with a linear gradient from 5 to $40 \%$ buffer 
A.

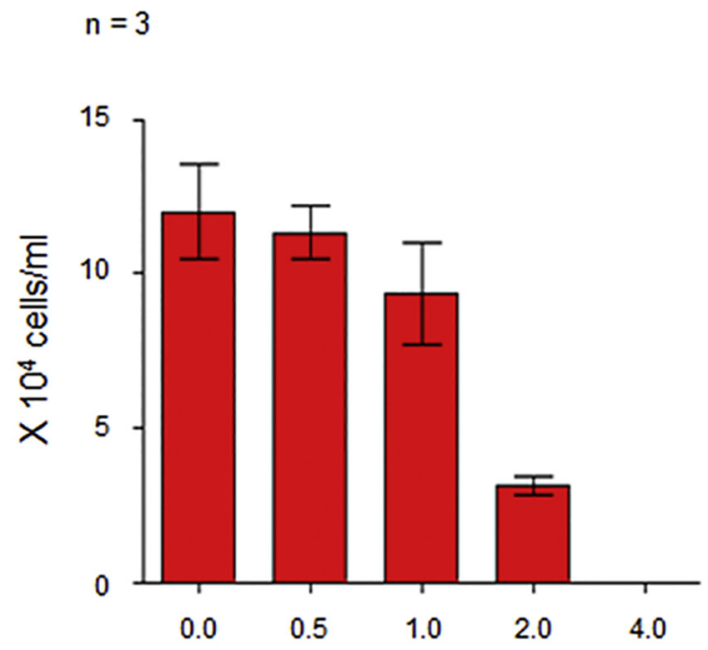

$h$ at $41^{\circ} \mathrm{C}$
B.

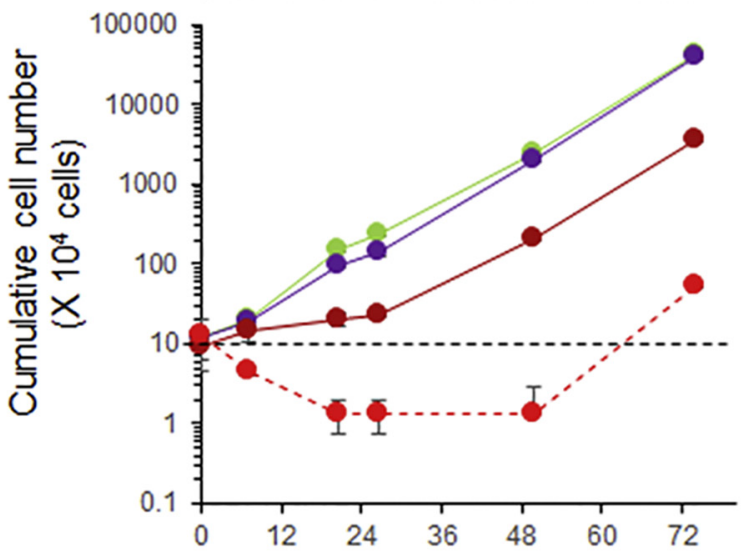

$\mathrm{h}$ after heat shock

C.

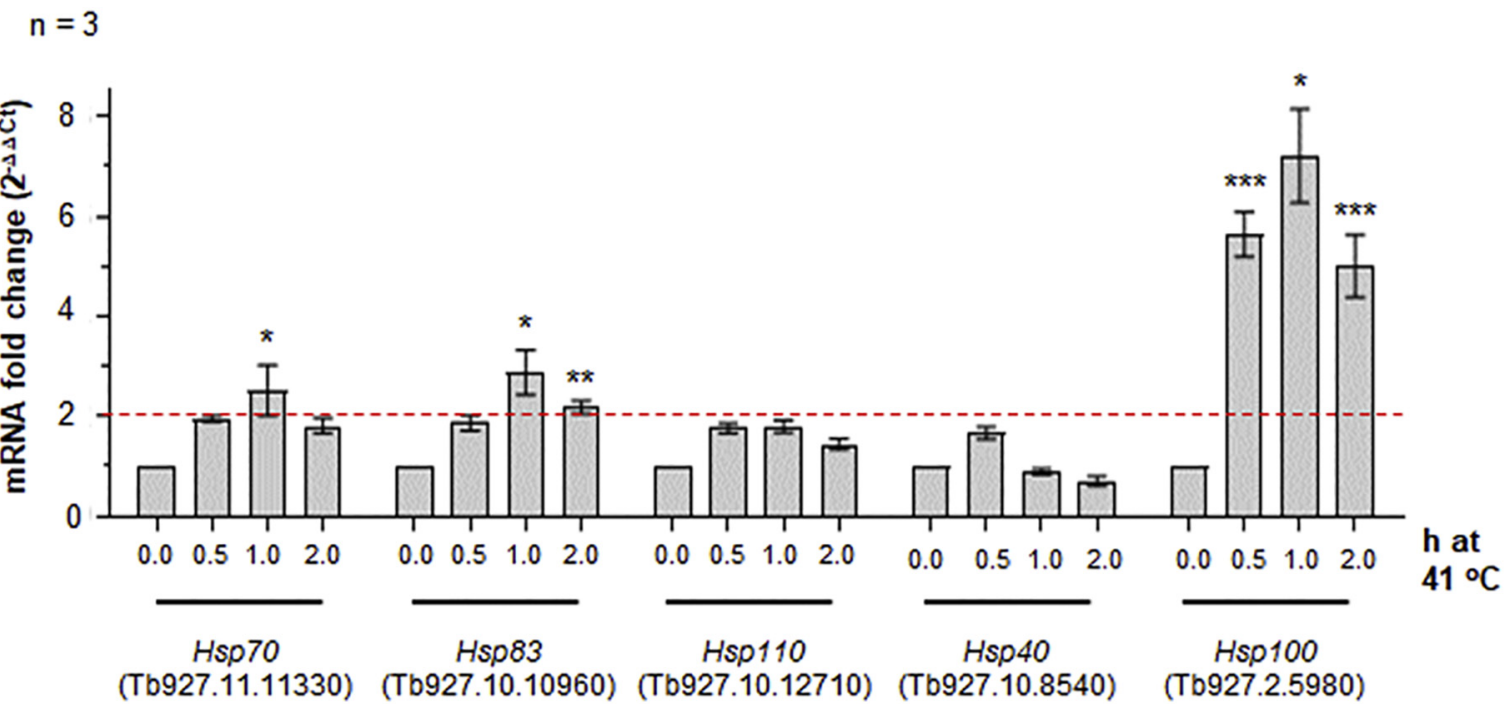

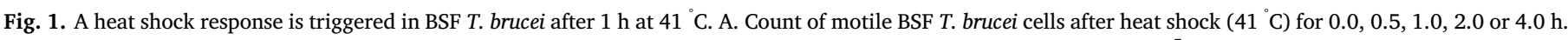

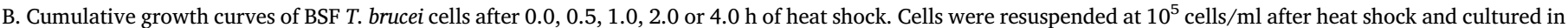

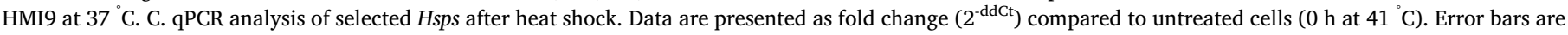

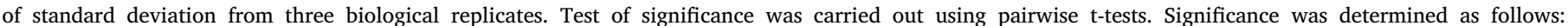
$*=\mathrm{P}<0.05 ;{ }^{* *}=\mathrm{P}<0.01 ; *^{* *}=\mathrm{P}<0.001$.

B over 65 min. The HPLC system was coupled to an LTQ Orbitrap Velos Elite mass spectrometer (Thermo Scientific) equipped with a Proxeon nanospray ion source. For phosphoproteomic analysis, the mass spectrometer was operated in data dependent mode to perform a survey scan over a range $335-1800 \mathrm{~m} / \mathrm{z}$ in the Orbitrap analyzer ( $R=60,000$ ), with each MS scan triggering fifteen $\mathrm{MS}^{2}$ acquisitions of the fifteen most intense ions using multistage activation on the neutral loss of 98 and 49 Thomsons in the LTQ ion trap [21]. For proteomic analysis, the mass spectrometer was operated in data dependent mode with each MS scan triggering fifteen $\mathrm{MS}^{2}$ acquisitions of the fifteen most intense ions in the LTQ ion trap. The Orbitrap mass analyzer was internally calibrated on the fly using the lock mass of polydimethylcyclosiloxane at $m / z 445.120025$.

\subsection{Mass spectrometry data processing}

Data was processed using MaxQuant [26] version 1.6.1.0 which incorporates the Andromeda search engine [27]. Proteins were identified by searching a protein sequence database containing $T$. brucei brucei 927 annotated proteins (Version 37, 11,074 protein sequences, TriTrypDB [28], http://www.tritrypdb.org/) supplemented with frequently observed contaminants (porcine trypsin, bovine serum albumins and mammalian keratins). Search parameters specified an MS tolerance of $6 \mathrm{ppm}$, an MS/MS tolerance at $0.5 \mathrm{Da}$ and full trypsin 
specificity, allowing for up to two missed cleavages. Carbamidomethylation of cysteine was set as a fixed modification and oxidation of methionine, $\mathrm{N}$-terminal protein acetylation and $\mathrm{N}$-pyroglutamate were allowed as variable modifications. The experimental design included matching between runs for the concatenated fractions in each experiment. Peptides were required to be at least 7 amino acids in length, with false discovery rates (FDRs) of 0.01 calculated at the levels of peptides, proteins and modification sites based on the number of hits against the reversed sequence database. Analysis of the phosphoproteomic data in MaxQuant $[26,27]$ used the same parameters, except for the addition of phosphorylation of serine, threonine and tyrosine residues as variable modifications. Phosphopeptides were identified with a $0.01 \mathrm{FDR}$, and phosphorylation sites were with only considered when the MaxQuant localisation probability $>0.75$.

SILAC ratios for phosphorylation sites were calculated using only data from the phosphoproteomic experiments, and SILAC ratios for proteins were calculated using only data from the proteomic experiments. SILAC ratios were calculated where at least one peptide could be uniquely mapped to a given protein group and required a minimum of two SILAC pairs. To account for any errors in the counting of the number of cells in each sample prior to mixing, the distribution of SILAC ratios was normalised within MaxQuant at the peptide level so that the median of $\log _{2}$ ratios is zero, as described by Cox et al. [26]. We have deposited the Thermo RAW files and search results in ProteomeXchange (http://www.proteomexchange.org) consortium via the Pride partner repository [29] with the dataset identifier PXD016482, enabling researchers to access the data presented here.

\subsection{Statistical analyses}

Statistical analyses of proteomics data was carried out using Perseus 1.6.1.3 [30] using data obtained from the function genomic database TriTrypDB [28]. Species that changed significantly upon HS were identified using intensity-weighted significance (Significance B) [26] with a Benjamini-Hochberg corrected FDR $\leq 0.01$. Categorical enrichment was determined using Fisher's exact test with a BenjaminiHochberg corrected FDR $\leq 0.01$. All other statistical analyses were carried out using GraphPad Prism 7.

\section{Results}

3.1. A heat shock response is triggered in BSF T. brucei after incubation at $41^{\circ} \mathrm{C}$

To simulate HS, BSF T. brucei were incubated at $41{ }^{\circ} \mathrm{C}$ for up to $4 \mathrm{~h}$, and cell viability immediately after heat treatment was determined by counting the cells that remained motile (Fig. 1A.). Although incubation for $1 \mathrm{~h}$ at $41{ }^{\circ} \mathrm{C}$ leads to a decrease in live cells it was not statistically significant ( $22 \%, \mathrm{p}=0.49$ ), and the number of live cells only decreased significantly after $2 \mathrm{~h}$ at $41{ }^{\circ} \mathrm{C}(74 \%, \mathrm{p}=0.04)$ and no motile cells were observed after $4 \mathrm{~h}$ at $41{ }^{\circ} \mathrm{C}$. To assess the recovery of cells from heat treatment, cells were returned to culture at $37^{\circ} \mathrm{C}$ and their growth monitored for $72 \mathrm{~h}$. As no motile cells were observable after $4 \mathrm{~h}$ of incubation at $41^{\circ} \mathrm{C}$, this treatment was omitted from this analysis. Cells incubated for $0.5 \mathrm{~h}$ at $41^{\circ} \mathrm{C}$ display a growth rate indistinguishable from untreated cells whilst cells incubated for $1 \mathrm{~h}$ at $41^{\circ} \mathrm{C}$ remain viable after HS, albeit with a reduced growth rate up to $24 \mathrm{~h}$ post-treatment, and cells incubated for $2 \mathrm{~h}$ at $41{ }^{\circ} \mathrm{C}$ continued to lose viability and failed to grow for up to $48 \mathrm{~h}$ post-treatment (Fig. 1B.).

To determine if a HS response was triggered in these cells, total RNA was harvested from heat-treated cells for qPCR quantitation of $H s p$ mRNA. Total RNA quality was assessed by electrophoresis on a $2 \%$ agarose gel, and only samples collected from cells incubated for $2 \mathrm{~h}$ at $41{ }^{\circ} \mathrm{C}$ showed signs of degradation (Fig. S1A.). All qPCR quantitation used the $28 \mathrm{~S} \beta$ subunit of ribosomal RNA as the reference and the critical threshold (Ct) value for this transcript remained constant with all HS treatments (Fig. S1B.). Five Hsps were selected for qPCR quantitation. These included two Hsps known to be up-regulated at the transcript level after HS in T. brucei [9,10] (Hsp70, Tb927.11.11330 and Hsp83, Tb927.10.10960), homologues to other members of the canonical Hsp70-Hsp40-Hsp110 network in yeast and mammals [31] (Hsp110, Tb927.10.12710 and Hsp40, Tb927.10.8540), and the closest T. brucei homologue to yeast Hsp104, which is also up-regulated after HS in PCF T. brucei [17,32-34] (Hsp100, Tb927.2.5980). Hsps with a $\geq 2$-fold increase were defined as up-regulated after heat shock. Consistent with previous reports [9,10], Hsp70 and Hsp83 transcript levels increase $>2$-fold after $1 \mathrm{~h}$ of HS (Fig. 1C.), indicating that a HS response is triggered in the treated cells. Likewise, Hsp100 transcript levels increase $\geq 6$-fold after HS, suggesting that the Hsp104 homologue has a role in the $T$. brucei HS response. The transcript level for other member of the Hsp70-Hsp40-Hsp110 network remain relatively unchanged ( $<2$-fold), which may indicate a divergence of the T. brucei HS response from that of its mammalian host.

\subsection{Quantitative phosphoproteomic and proteomic analysis of heat shock sensing in BSF T. brucei}

To dissect the HS response in BSF T. brucei in an unbiased manner, we conducted a SILAC-based quantitative proteomic and phosphoproteomic analysis of the BSF T. brucei heat shock response. We chose to examine an early time point $\left(41^{\circ} \mathrm{C}\right.$ for $1 \mathrm{~h}$ ) to capture signal transduction events involved in sensing and responding to heat shock, as at this time point cells clearly display a temporary growth phenotype (Fig. 1B). Cells were grown in HMI11-SILAC supplemented with $10 \%$ dialysed FCS and either normal isotopic abundance L-arginine and Llysine (Light) or ${ }^{13} \mathrm{C}_{6},{ }^{15} \mathrm{~N}_{4}$ L-arginine and ${ }^{13} \mathrm{C}_{6},{ }^{15} \mathrm{~N}_{2}$ L-lysine (Heavy) for $>9$ cell divisions to ensure steady state labelling, and then the Light cells were heat shocked at $41{ }^{\circ} \mathrm{C}$ for $1 \mathrm{~h}$. Heavy and Light cells were independently hypotonically lysed in the presence of phosphatase and protease inhibitors, then equal cell numbers $\left(\sim 2 \times 10^{8}\right)$ combined and subjected to FASP and tryptic digest [24,25]. Phosphopeptides were separated from peptides using Fe-IMAC [19], and the two pools separately fractionated using the high $\mathrm{pH}$ reverse phase with concatenation prior to LC-MS/MS analysis [20].

Analysis of the phosphoproteomic data identified a total of 8055 phosphorylation sites (localisation probability $\geq 0.75$ ) on 2381 proteins, and was able to quantify the change in abundance between the HS and untreated cells for 7258 phosphorylation sites on 2137 proteins (Supplementary Table S2). A total of 193 of phosphorylation sites on 148 proteins changed abundance significantly upon HS (FDR $\leq 0.01$ ), with 138 sites increasing in abundance with an average 6-fold increase and 55 sites decreasing in abundance by an average of 4-fold (Fig. 2A, Supplementary Table S3). Proteomic analysis identified a total of 4002 proteins, and was able to quantify the change in abundance of 2581 proteins between the HS and untreated cells (Supplementary Table S4). The overall changes in protein abundance were modest, with only 20 proteins showing significant changes (FDR $<0.01$ ), with 7 proteins increasing in abundance by an average of 1.4 -fold and 13 proteins decrease in abundance by an average of 1.5 -fold (Fig. 2B, Supplementary Table S4).

The 193 HS sensitive phosphorylation sites showed significant categorical enrichment (FDR $\leq 0.01$ ) for 16 curated Gene Ontology (GO) terms (Fig. 3). The enriched GO terms include many with anticipated roles in HS response included RNA Helicase activity (FDR $5.9 \times 10^{-7}$ ), P-body $\left(\right.$ FDR $\left.=6.8 \times 10^{-6}\right)$, eIF4-F complex $\left(\right.$ FDR $\left.=6.6 \times 10^{-6}\right)$, nuclear and cytosolic stress granules (FDR $=9.82 \times 10^{-6}$ and $3.5 \times 10^{-}$ $\left.{ }^{4}\right)$ and mRNA binding $\left(\right.$ FDR $\left.=7.0 \times 10^{-4}\right)$. In addition, there was significant enrichment for known and novel RBPs (FDR $2.7 \times 10^{-7}$ ) $[35,36]$, and some enrichment $($ FDR $\leq 0.05)$ of HSPs $($ FDR $=0.013)$ and Protein kinases (FDR $=0.011$ ) $[37,38]$.

Amongst the 20 proteins that changed in abundance in response to HS there was no significant categorical enrichment (FDR $\leq 0.01$ ) of GO 
A.

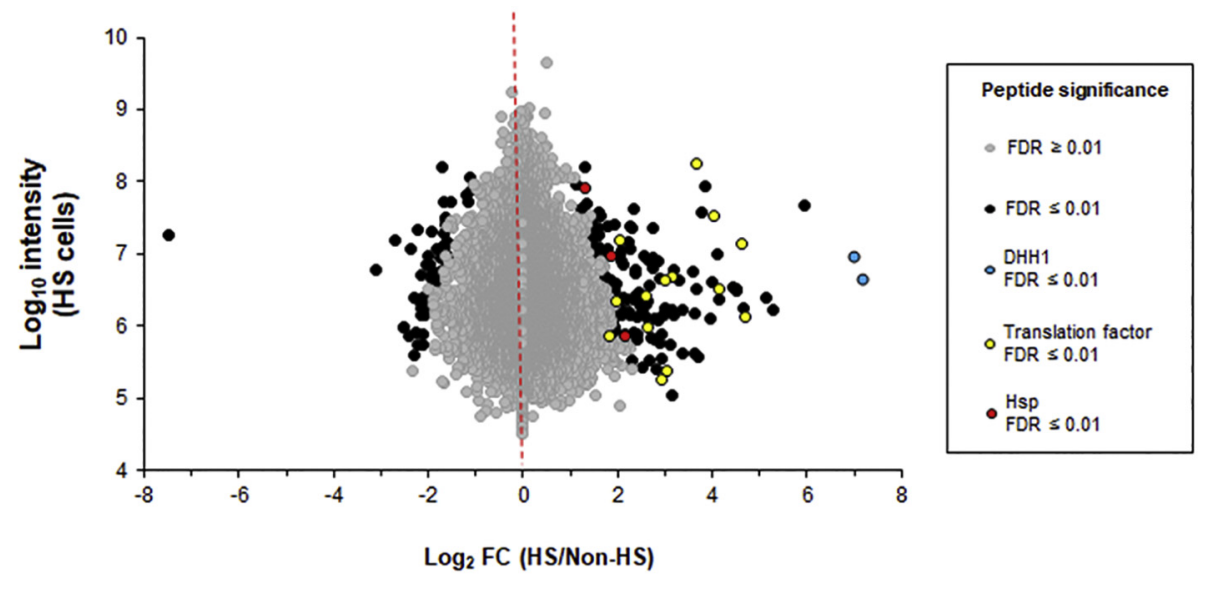

B.

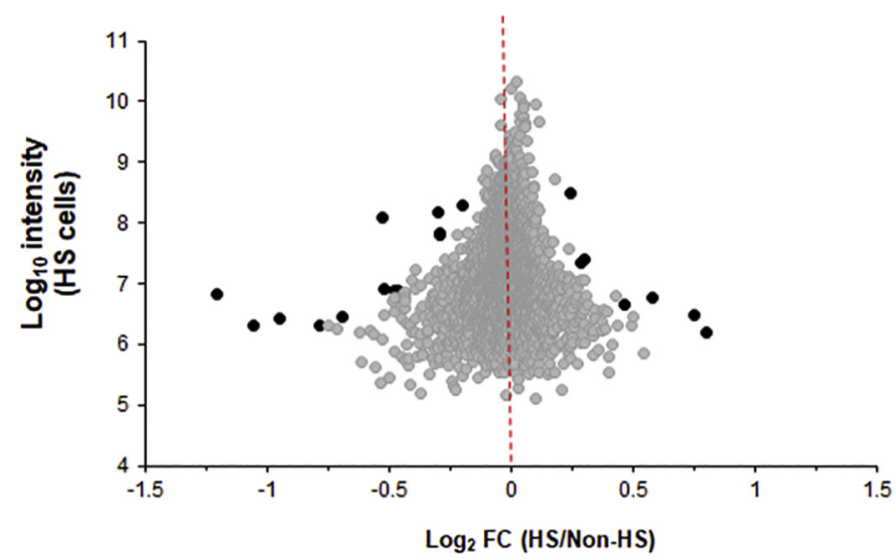

Protein significance

FDR $\geq 0.01$

- FDR $\leq 0.01$

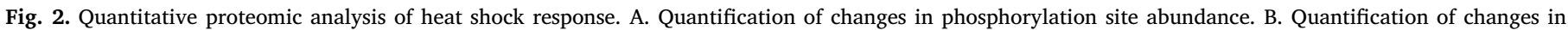

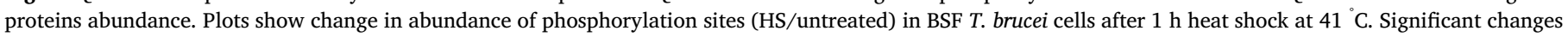
(FDR $\leq 0.01)$ following heat shock are indicated as black circles. DHH1 - blue, Translation factors - yellow, Hsps - red.

terms, and only HSPs were significant enriched (FDR $=0.0078$ ). Interestingly, the abundance of Hsp100 was significantly increased (1.2-fold) in agreement with our qPCR data ( $\geq 6$-fold, Fig. 1C), consistent with a more rapid response occurring at the mRNA level than protein level. These data indicate that protein abundance does not rapidly respond ( $\leq 1 \mathrm{~h}$ ) to heat shock, and that the changes observed are smaller and less widespread than changes in phosphorylation site abundance.

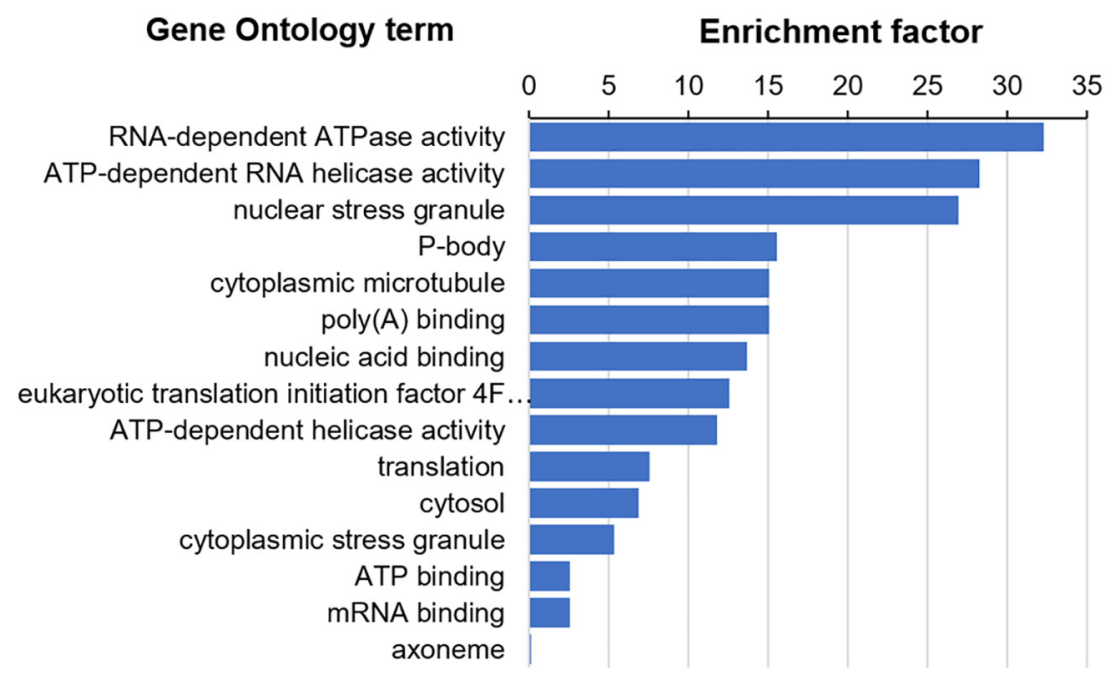

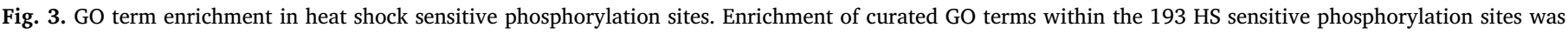
determined using Fisher's exact test with a Benjamini-Hochberg FDR $\leq 0.01$. 

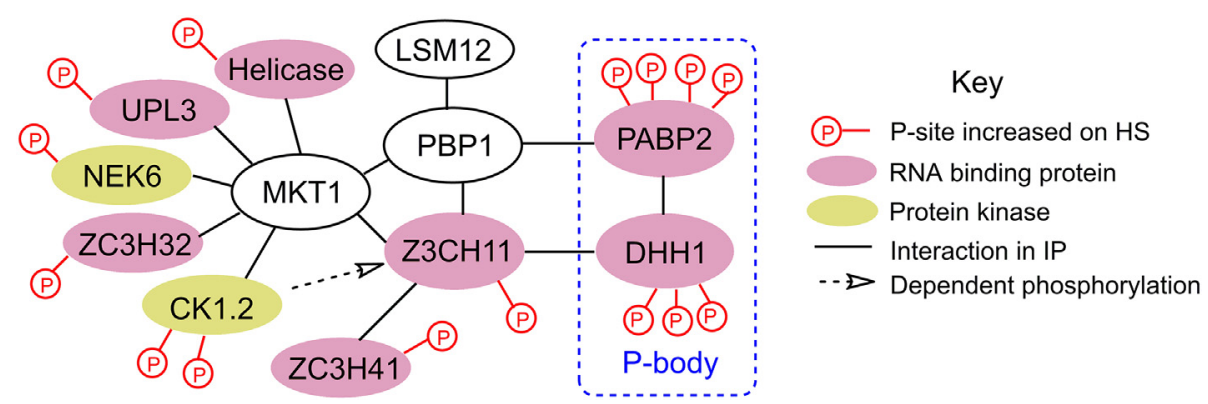

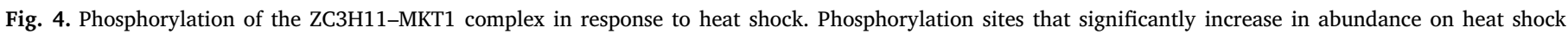
$($ FDR $<0.01)$ are shown. Interaction data is taken from Singh et al [42].

\subsection{Phosphorylation modulates the formation of P-bodies and the post- transcriptional regulation of heat shock}

The largest increases in phosphorylation site abundance occur on the DEAD-box RNA helicase DHH1 S84 (140-fold) and T82 (126-fold), with an additional site T22 showing a more modest but significant increase (5-fold). In PCF T. brucei DHH1 represses certain life cycle stage specific mRNAs via recognition of an AU rich element in the 3' UTR $[39,40]$. Expression of an ATPase deficient DHH1 mutant in PCF increases the number of P-bodies and selectively stabilises certain mRNAs, leading to ISG75 protein accumulation, which we also observe to be significantly increased in abundance upon heat shock (1.4-fold). Upon HS DHH1 is known to re-localise to P-bodies, where it is largely but not entirely co-localised with PAPB2 but not PAPB1 [41]. Phosphorylation of PABP2 was also significantly increased on HS at S93, T167, S212 and S259 by 8- to 16-fold in our data set, whilst phosphorylation of PABP1 was not significantly changed. These data suggest that phosphorylation of DHH1 and PABP2 may play an important role in their re-localisation to P-bodies in response to HS.

DHH1 and PABP2 have previously been shown to co-immunoprecipitate with the CCCH zinc-finger ZC3H11 (Fig. 4) [42], a key regulator of HS response that is essential in BSF but not PCF T. brucei [17]. The abundance of ZC3H11 is known to increase after HS [43], and the protein has been shown to bind to and stabilise the mRNA of many chaperones and Hsps including Hsp70, Hsp83 and Hsp100 [17], in agreement with our qPCR data (Fig. 1C). We observe a significant increase in phosphorylation of ZC3H11 S275 (16-fold) upon HS, along with three other unquantified phosphorylation sites S23, S26 and S279 that were only observed under HS conditions (localisation $>0.95$ ). ZC3H11 has previously been reported to be phosphorylated based on changes in protein migration on a gel after phosphatase treatment $[17,43]$, but the specific modification sites were not previously determined. We were only able to observe the protein ( 5 unique peptides) after HS, supporting an increase in abundance, but were unable to quantify the increase in abundance at the protein level. ZC3H11 forms a complex with MKT1, LSM12 and the PABP-binding protein PBP1 [42], but we did not observe significant changes to these proteins at the protein or phosphorylation site level upon HS. MKT1 associates with NEK6 (Tb927.5.2820) and CK1.2 (Tb927.5.800), and it has been shown that RNAi of CK1.2 reduces the phosphorylation of ZC3H11 under normal and heat shock conditions, whereas RNAi of NEK6 had no effect on ZC3H11 expression [43]. We observe significant increases in phosphorylation of NEK6 S27 (8-fold) and two sites on CK1.2 S19 and S21 (2.8- and 3.5-fold respectively). We also observed significant increases in phosphorylation site abundance on a $\mathrm{CCCH}$ zinc-finger protein ZC3H41 that associates with Z3CH11 as well as a DEAD box Helicase (Tb927.3.260), a ubiquitin ligase with a CCCH zinc-finger UPL3 (Tb927.8.1590) and a CCCH zinc-finger proteins ZC3H32 that associate with MKT1 [42] (Fig. 4). Taken together, these data suggest that the ZC3H11-MKT1 complex is an important signalling node that is dynamically phosphorylated to modulate RNA binding and P-body formation in response to heat shock.

\subsection{Phosphorylation of heat shock proteins occurs more rapidly than changes in abundance}

Phosphorylation site on three Hsps were significantly increased after HS: Hsp70.c S464 (Tb927.11.11290, 4.5-fold), Hsp83 S48 (Tb927.10.10960, 3.7-fold) and Hsp70 S79 (Tb927.11.11330, 2.5-fold). None of these three Hsps showed a significant change in protein abundance, but Hsp70 and Hsp83 were shown to have $>2$-fold increase at the mRNA level after HS (Fig. 1C). Phosphorylation sites on a further three Hsps (Hsp110, Tb927.10.12710; Hsp84, Tb927.11.250; Hsp83, Tb927.10.10960) were changed > 2-fold upon HS, but were not considered to be significantly changes in our analysis $(0.01>$ FDR $>$ 0.05 ), and did not change at the protein level. At the protein level only Hsp100 (Tb927.2.5980) was significantly increased (1.2-fold), in agreement with our qPCR data (Fig. 1C).

\subsection{Translation factors are phosphorylated in response to heat shock}

We found significant differential phosphorylation at 18 sites on 9 different proteins that are either eukaryotic translation initiation factors or have an established role in translation (Table 1). The T. brucei genome has an expanded complement of components of the eIF4F capdependent initiation complex subunits with two eIF4A, six eIF4E and five eIF4G variants, providing an opportunity for greater complexity in translational control. The eukaryotic translation initiation factor $4 \mathrm{~F}$ complex in T. brucei consists of eIF4A-1-eIF4E-4-eIF4G-3 which is important for the G1/S transition [44], and a second eIF4A-1-eIF4E3-eIF4G-3/4 complex that may regulate the G2/M transition [20]. HS induced a significant increase in phosphorylation on eIF4A-1 at S51, S101, S147, T155, S237 and T325 by between 3-fold and 24-fold respectively, and a decrease in phosphorylation on eIF4E-4 S90 by 3-fold. Phosphorylation also increases on eIF4G4 S23 4-fold and S68 6-fold, and on Translation associated element 2 S502 (Tb927.11.13060) by 25fold, proteins shown to interact and localise to stress granules upon starvation [45]. We did not observe any significant change to the phosphorylation of eIF2 $\alpha$ (Tb927.3.2900), consistent with reports that HS acts independently of eIF $2 \alpha$ T169 phosphorylation (equivalent to serine 51 in mammalian eIF2 $\alpha$ ) [9]. Interestingly, the MKT1-like protein MKT1L S1005 (Tb927.10.1490) has the second largest decrease (8.5-fold) in phosphorylation site abundance, and in Leishmania tarentolae MKT1L co-purifies with the spliceosome complex responsible for trans-splicing a common spliced leader sequence to all mRNAs [46]. Phosphorylation of proteins involved in translational complexes is suggestive of a mechanism which contributes to the repression of translation and the formation of stress granules/P-bodies in response to HS. 
Table 1

Heat shock alters the phosphorylation of translational machinery.

\begin{tabular}{|c|c|c|c|}
\hline GeneDB & Name & Phosphorylation site & Fold-change \\
\hline \multirow[t]{6}{*}{ Tb927.9.4680 } & eIF4A-1 & S51 & 24-fold up \\
\hline & & S101 & 17-fold up \\
\hline & & S147 & 3.5-fold up \\
\hline & & T155 & 3.0-fold up \\
\hline & & S237 & 13-fold up \\
\hline & & T325 & 7.7-fold up \\
\hline Tb927.6.1870 & eIF4E-4 & S90 & 3.1-fold down \\
\hline Tb927.11.11770 & eIF4E-3 & S424 & 3.5-fold up \\
\hline \multirow[t]{2}{*}{ Tb927.11.10560 } & eIF4G-4 & S23 & 3.9-fold up \\
\hline & & S68 & 6.3-fold up \\
\hline Tb927.8.4500 & eIF4G-5 & S15 & 4.1-fold up \\
\hline Tb927.11.13060 & Translation associated element 2 & S502 & 26.2-fold up \\
\hline Tb927.11.9610 & Translation initiation factor $3 \mathrm{i}$ & S248 & 5.8-fold up \\
\hline Tb927.11.2300 & Eukaryotic peptide chain release factor subunit 1 & S172 & 7.1-fold up \\
\hline \multirow[t]{4}{*}{ Tb927.9.10770 } & РАBP2 & s93 & 16-fold up \\
\hline & & T167 & 8.2-fold up \\
\hline & & S212 & 8.8-fold up \\
\hline & & S259 & 8.1-fold up \\
\hline
\end{tabular}

\subsection{Phosphorylation of protein kinases in response to heat shock}

The T. brucei genome contains between 176 and 182 putative protein kinases (PKs) [37,38], and our data contains 18 phosphorylation sites on 14 PKs that alter significantly in response to heat shock (Table 2), whilst no PKs change significantly at the protein level. PKs with HS sensitive phosphorylation sites include many that have been demonstrated to be essential, and where RNAi ablation in the bloodstream form causes a cell cycle defect, including CK1.2, GSK3, MAPK6, PLK, RCK [47-50]. Amongst the 18 significantly regulated phosphorylation sites, 8 sites decrease in abundance upon HS including the essential AGC S229 (3.2-fold), PLK T198 (3.6-fold) and CDK2 Y57 (3.6fold). The decrease in phosphorylation of PLK T198 on the T-loop will reduce its activity, preventing progression through the cell cycle as PLK activity is required for cytokinesis. Amongst the 10 sites that show an increase in phosphorylation are GSK3 S44 (4.3-fold) and NEK17 S197 (5.7-fold). Phosphorylation of mammalian GSK3 on S9 reduces its catalytic activity, and it is possible that the $S 44$ phosphorylation adjacent to the N-terminus of the T. brucei GSK3 kinase domain has a similar inhibitory role. NEK17 has been identified as being involved in the stumpy induction pathway, with a potential role in cell cycle exit and quiescence [51]. Interestingly, our data contains only 3 phosphorylation sites on 2 protein phosphatases that alter significantly in response to heat shock (PP2A Tb927.9.6150 S91 12-fold up and S44 9.8-fold up; Tb927.10.1620 S2244 9.7-fold up), whilst only one

Table 2

Phosphorylation of protein kinases in response to heat shock.

\begin{tabular}{llll}
\hline GeneDB & Name & Phosphorylation site & Fold change \\
\hline Tb927.3.2440 & AGC Essential & S229 & 3.2-fold down \\
Tb927.10.5310 & AMPK & T464 & 3.9-fold down \\
& & S468 & 4.0-fold down \\
Tb927.10.3900 & CAMK/CAMKL & S167 & 5.3-fold down \\
Tb927.5.800 & CK1.2 & S21 & 3.5-fold up \\
& & S19 & 2.8-fold up \\
Tb927.10.3340 & CMGC/MAPK & T155 & 3.1-fold down \\
& & T844 & 4.0-fold down \\
Tb927.7.960 & CMGC/SRPK & S68 & 7.2-fold up \\
Tb927.7.7360 & CRK2 & Y57 & 3.6-fold down \\
Tb927.10.13780 & GSK3 short & S44 & 4.4-fold up \\
Tb927.8.3550 & MAPK3 & Y195 & 4.1-fold up \\
Tb927.10.5930 & NEK17 & S197 & 5.8-fold up \\
Tb927.5.2820 & NEK6 & S27 & 8.1-fold up \\
Tb927.7.6310 & PLK & T198 & 3.6-fold down \\
Tb927.3.690 & RCK & Y177 & 3.0-fold up \\
Tb927.1.1530 & STE group & S731 & 5.7-fold up \\
\hline
\end{tabular}

changed significantly at the protein level (Tb927.10.1740, 1.7-fold down).

\section{Discussion}

Our quantitative proteomic and phosphoproteomic analysis shows that during the first hour of HS there is little change in overall protein abundance, but nearly 200 phosphorylation sites are significantly changed in abundance. In response to HS we observe significantly different phosphorylation on RBPs, PKs, translational components, and Pbody / stress granule proteins. The decreased phosphorylation of protein kinases with established roles in the cell cycle and increased phosphorylation of translational components such as the eiF4F complex suggests that these phosphorylation sites may play a role in the global translational arrest and exit from the cell cycle that occurs upon HS. Significant changes in abundance was observed for only 20 proteins, suggesting that the $1 \mathrm{~h}$ time point is too early to detect changes at the protein level despite evidence of upregulation of Hsp at the mRNA level and altered phosphorylation of the global translational machinery.

The large increase in phosphorylation site abundance on DHH1 and PABP2 may have a role in their sequestration into P-bodies/stress granules in response to heat shock [41]. Both DHH1 and PABP2 have been shown to associate with the ZC3H11-MKT1 complex, along with a number of other RBPs and PKs including CK1.2 that are dynamically phosphorylated in response to HS [42], suggesting the complex may represent a signal integration node. Following HS, the half-life of both phosphorylated and dephosphorylated ZC3H11 increases, resulting in an increase in the abundance of target mRNAs for Hsps and chaperone proteins $[17,43]$. The stability of the dephosphorylated form is greater, and accumulates with prolonged HS. Phosphorylation of ZC3H11 in vitro is dependent on the activity of CK1.2, and in vitro CK1.2 can phosphorylate N-terminal portions of ZC3H11, but the sites of phosphorylation have not previously been determined [43]. We observed that phosphorylation of ZC3H11 S275 increases 16-fold upon HS, and phosphorylation at S23, S26 and S279 was observed only after HS conditions but we did not observe any sites that occurred only in untreated cells. Of the identified phosphorylation sites, only S23 and S26 occur in the portion of ZC3H11 used in the recombinant kinase assay, and none occur within canonical CK1 recognition motifs, suggesting that the phosphorylation of ZC3H11 by CK1.2 may not be direct. The activity of CK1.2 against ZC3H11 in IP experiments was reduced when cell were treated at $41{ }^{\circ} \mathrm{C}$ for $1 \mathrm{~h}$ [43], and we observe an $\sim 3$-fold increase in phosphorylation of CK1.2 at S19 and S21 after HS that may be responsible for the decreased activity. Further experiments are required to examine both the temporal dynamic of these phosphorylation 
sites and to clarify their effect on the HS response.

In mammalian cells, but not yeast and fruit flies, the HS translation arrest is triggered by the phosphorylation of the translation factor eIF $2 \alpha$ which blocks translation initiation $[9,12,13]$. We did not observe any significant change to the phosphorylation of eIF2 $\alpha$, consistent with reports that HS acts independently of eIF $2 \alpha$ T169 phosphorylation [9], suggesting the presence of an eIF2 $\alpha$-independent mechanism of translation arrest. The differential phosphorylation of eIF4A-1, eIF4E-4, and eIF4G4 in response to HS suggests these components of the eIF4F capdependent initiation complexes are involved in an atypical mechanism of HS translation arrest in T. brucei.

T. brucei lacks the unfolded protein response (UPR) found in most eukaryotes, but ER stress induces the alternative spliced leader silencing (SLS) pathway that prevents the trans-splicing of a common spliced leader (SL) sequence essential for mRNA maturation [52]. Indeed, HS has been previously reported to disrupt the trans-splicing of tubulin genes, but not Hsp70 and Hsp85 [10], suggesting a role for selective SLS in HS response. The observed decrease in phosphorylation of a MKT1L, a putative component of the spliceosome complex, is amongst the largest we detect, supporting a role for SLS in the global translational repression triggered by HS. However, our proteomic analysis does not detect either PK3 or TRF4 at the protein or phosphorylation site level, two key proteins involved in activation of SLS [53].

Hsps bind to exposed hydrophobic regions on misfolded proteins to stabilise or promote degradation. Five major groups are conserved across all organisms (Hsp60s, Hsp70s, Hsp90s, Hsp100s and small Hsps), and some have defined functions in eukaryotes. In yeast and mammals, the canonical Hsp70-Hsp40-Hsp110 network functions to disaggregate misfolded proteins following HS and labels them for ubiquitination and degradation [31], and Hsp104 forms a hexameric ring which threads misfolded proteins through its central pore to resolve protein aggregates that form following HS [32-34]. In response to $1 \mathrm{~h}$ HS at $41{ }^{\circ} \mathrm{C}$ we observe a significant increases in the mRNA levels of Hsp70, Hsp83 and Hsp100, but not other members of the Hsp70-Hsp40Hsp110 network. Only two heat shock proteins change in abundance at the protein level significantly (Hsp100 increased 1.2-fold, Hsp70.a decreased 1.2-fold) whilst phosphorylation of Hsp70, Hsp70.c and Hsp83 increased 2.5-4.5-fold. However, further proteomic time points are required to determine if the magnitude of changes increases with prolonged HS or are maintained during recovery. Differential phosphorylation of Hsps but not changes to mRNA or protein level has been observed in the HS response of Leishmania donovani [54-56], a closely related kinetoplastid parasite, where Hsps and protein chaperones including Hsp70, Hsp90 and the Hsp complex protein STI1 are phosphorylated following HS. It has been suggested that the functions of these HS-associated proteins in Leishmania are phosphorylation-dependent, though this has only been experimentally demonstrated for STI1 [54-56].

Temperature changes in the host are a major physiological challenge to parasites and factors conferring HS tolerance constitute overlooked virulence factors. A better understanding of these virulence factors would pave the way for the development of novel drug therapies which selectively target $T$. brucei. This can be achieved either by the development of compounds which directly target Hsps, or via inhibitors of phosphorylation that could specifically target the activation of the HS response.

\section{Conclusions}

Quantitative proteomic and phosphoproteomic analysis of the heat shock response in BSF T. brucei has revealed that protein abundance does not rapidly respond ( $\leq 1 \mathrm{~h}$ ) to heat shock, but that significant changes in phosphorylation site abundance occur. We have identified dynamic phosphorylation occurring on RBPs and PKs that may play a role in translational arrest, exit from the cell cycle, P-body formation and upregulation of Hsp and chaperones. This is unlike the response in mammalian cells where translation arrest is triggered by eIF $2 \alpha$ phosphorylation. Finally, the ZC3H11-MKT1 post-transcriptional regulatory complex appears to represent a key signal integration node in the heat shock response.

Supplementary data to this article can be found online at https:// doi.org/10.1016/j.jprot.2020.103735.

\section{Acknowledgements}

The authors would like to acknowledge Calvin Tiengwe for qPCR reagents and discussions. The authors also wish to thank Matthew Child, Paul McKean, Philippe Bastin and Brice Rotureau for helpful discussions. MDU and CB were supported by a BBSRC New Investigator Award (BB/M009556/1) and CPO is employed by the Wellcome Trust (grant no. 095161).

\section{References}

[1] A.P. Shaw, G. Cecchi, G.R. Wint, R.C. Mattioli, T.P. Robinson, Mapping the economic benefits to livestock keepers from intervening against bovine trypanosomosis in Eastern Africa, Prev. Vet. Med. 113 (2) (2014) 197-210.

[2] P.P. Simarro, G. Cecchi, J.R. Franco, M. Paone, A. Diarra, J.A. Ruiz-Postigo, E.M. Fevre, R.C. Mattioli, J.G. Jannin, Estimating and mapping the population at risk of sleeping sickness, PLoS Negl. Trop. Dis. 6 (10) (2012) e1859.

[3] N. Baker, H.P. De Koning, P. Maser, D. Horn, Drug resistance in African trypanosomiasis: The melarsoprol and pentamidine story, Trends Parasitol. 29 (3) (2013) $110-118$.

[4] R. Ross, D. Thomson, A case of sleeping sickness showing regular periodical increase if the parasite disclosed, Br. Med. J. 1 (2582) (1910) 1544-1545.

[5] A. Sinha, C. Grace, W.K. Alston, F. Westenfeld, J.H. Maguire, African trypanoso miasis in two travelers from the United States, Clin. Infect. Dis. 29 (4) (1999) $840-844$.

[6] J.W. Magona, J. Walubengo, W. Olaho-Mukani, N.N. Jonsson, M.C. Eisler, Diagnostic value of rectal temperature of African cattle of variable coat colour infected with trypanosomes and tick-borne infections, Vet. Parasitol. 160 (3-4) (2009) 301-305.

[7] J.R. Franco, P.P. Simarro, A. Diarra, J.G. Jannin, Epidemiology of human African trypanosomiasis, Clin. Epidemiol. 6 (2014) 257-275.

[8] C.R. Taylor, C.P. Lyman, Heat storage in running antelopes: Independence of brain and body temperatures, Am. J. Physiol. 222 (1) (1972) 114-117.

[9] S. Kramer, R. Queiroz, L. Ellis, H. Webb, J.D. Hoheisel, C. Clayton, M. Carrington, Heat shock causes a decrease in polysomes and the appearance of stress granules in trypanosomes independently of eIF2(alpha) phosphorylation at Thr169, J. Cell Sci. 121 (Pt 18) (2008) 3002-3014.

[10] M.L. Muhich, M.P. Hsu, J.C. Boothroyd, Heat-shock disruption of trans-splicing in trypanosomes: Effect on Hsp70, Hsp85 and tubulin mRNA synthesis, Gene 82 (1) (1989) 169-175.

[11] C. Chakraborty, C. Clayton, Stress susceptibility in Trypanosoma brucei lacking the RNA-binding protein ZC3H30, PLoS Negl. Trop. Dis. 12 (10) (2018) e0006835.

[12] T. Grousl, P. Ivanov, I. Frydlova, P. Vasicova, F. Janda, J. Vojtova, K. Malinska, I. Malcova, L. Novakova, D. Janoskova, L. Valasek, J. Hasek, Robust heat shock induces eIF2alpha-phosphorylation-independent assembly of stress granules containing EIF3 and 40 S ribosomal subunits in budding yeast, Saccharomyces cerevisiae, J. Cell Sci. 122 (Pt 12) (2009) 2078-2088.

[13] N.G. Farny, N.L. Kedersha, P.A. Silver, Metazoan stress granule assembly is mediated by P-eIF2alpha-dependent and -independent mechanisms, RNA 15 (10) (2009) 1814-1821.

[14] C.C. Avila, L. Peacock, F.C. Machado, W. Gibson, S. Schenkman, M. Carrington, B.A. Castilho, Phosphorylation of eIF2alpha on Threonine 169 is not required for Trypanosoma brucei cell cycle arrest during differentiation, Mol. Biochem. Parasitol. 205 (1-2) (2016) 16-21.

[15] D.B. Mahat, H.H. Salamanca, F.M. Duarte, C.G. Danko, J.T. Lis, Mammalian heat shock response and mechanisms underlying its genome-wide transcriptional regulation, Mol. Cell 62 (1) (2016) 63-78.

[16] C. Clayton, The regulation of trypanosome gene expression by RNA-binding proteins, PLoS Pathog. 9 (11) (2013) e1003680.

[17] D. Droll, I. Minia, A. Fadda, A. Singh, M. Stewart, R. Queiroz, C. Clayton, Posttranscriptional regulation of the trypanosome heat shock response by a zinc finger protein, PLoS Pathog. 9 (4) (2013) e1003286.

[18] A. Singh, I. Minia, D. Droll, A. Fadda, C. Clayton, E. Erben, Trypanosome MKT1 and the RNA-binding protein ZC3H11: interactions and potential roles in post-transcriptional regulatory networks, Nucleic Acids Res. 42 (7) (2014) 4652-4668.

[19] B. Ruprecht, H. Koch, G. Medard, M. Mundt, B. Kuster, S. Lemeer, Comprehensive and reproducible phosphopeptide enrichment using iron immobilized metal ion affinity chromatography (Fe-IMAC) columns, Mol. Cell Proteomics 14 (1) (2015) 205-215.

[20] C. Benz, M.D. Urbaniak, Organising the cell cycle in the absence of transcriptional control: Dynamic phosphorylation co-ordinates the Trypanosoma brucei cell cycle post-transcriptionally, PLoS Pathog. 15 (12) (2019) e1008129.

[21] M.J. Schroeder, J. Shabanowitz, J.C. Schwartz, D.F. Hunt, J.J. Coon, A neutral loss 
activation method for improved phosphopeptide sequence analysis by quadrupole ion trap mass spectrometry, Anal. Chem. 76 (13) (2004) 3590-3598.

[22] K.J. Livak, T.D. Schmittgen, Analysis of relative gene expression data using realtime quantitative PCR and the 2(-Delta Delta C(T)) method, Methods 25 (4) (2001) 402-408.

[23] M.R. Domingo-Sananes, B. Szoor, M.A. Ferguson, M.D. Urbaniak, K.R. Matthews, Molecular control of irreversible bistability during trypanosome developmental commitment, J. Cell Biol. 211 (2) (2015) 455-468.

[24] M.D. Urbaniak, D.M. Martin, M.A. Ferguson, Global quantitative SILAC phosphoproteomics reveals differential phosphorylation is widespread between the procyclic and bloodstream form lifecycle stages of Trypanosoma brucei, J. Proteome Res. 12 (5) (2013) 2233-2244.

[25] J.R. Wisniewski, A. Zougman, N. Nagaraj, M. Mann, Universal sample preparation method for proteome analysis, Nat. Methods 6 (5) (2009) 359-362.

[26] J. Cox, M. Mann, MaxQuant enables high peptide identification rates, individualized p.p.b.-range mass accuracies and proteome-wide protein quantification, Nat. Biotechnol. 26 (12) (2008) 1367-1372.

[27] J. Cox, N. Neuhauser, A. Michalski, R.A. Scheltema, J.V. Olsen, M. Mann, Andromeda: a peptide search engine integrated into the MaxQuant environment, J. Proteome Res. 10 (4) (2011) 1794-1805.

[28] M. Aslett, C. Aurrecoechea, M. Berriman, J. Brestelli, B.P. Brunk, M. Carrington, D.P. Depledge, S. Fischer, B. Gajria, X. Gao, M.J. Gardner, A. Gingle, G. Grant, O.S. Harb, M. Heiges, C. Hertz-Fowler, R. Houston, F. Innamorato, J. Iodice, J.C. Kissinger, E. Kraemer, W. Li, F.J. Logan, J.A. Miller, S. Mitra, P.J. Myler, V. Nayak, C. Pennington, I. Phan, D.F. Pinney, G. Ramasamy, M.B. Rogers, D.S. Roos, C. Ross, D. Sivam, D.F. Smith, G. Srinivasamoorthy, C.J. Stoeckert Jr., S. Subramanian, R. Thibodeau, A. Tivey, C. Treatman, G. Velarde, H. Wang, TriTrypDB: A functional genomic resource for the Trypanosomatidae, Nucleic Acids Res. 38 (Database issue) (2010) D457-D462.

[29] J.A. Vizcaino, R.G. Cote, A. Csordas, J.A. Dianes, A. Fabregat, J.M. Foster, J. Griss, E. Alpi, M. Birim, J. Contell, G. O’kelly, A. Schoenegger, D. Ovelleiro, Y. PerezRiverol, F. Reisinger, D. Rios, R. Wang, H. Hermjakob, The PRoteomics IDEntifications (PRIDE) database and associated tools: status in 2013, Nucleic Acids Res. 41 (Database issue) (2013) D1063-D1069.

[30] S. Tyanova, T. Temu, P. Sinitcyn, A. Carlson, M.Y. Hein, T. Geiger, M. Mann, J. Cox, The Perseus computational platform for comprehensive analysis of (prote)omics data, Nat. Methods 13 (9) (2016) 731-740.

[31] B. Chen, M. Retzlaff, T. Roos, J. Frydman, Cellular strategies of protein quality control, Cold Spring Harb. Perspect. Biol. 3 (8) (2011) a004374.

[32] R. Lum, J.M. Tkach, E. Vierling, J.R. Glover, Evidence for an unfolding/threading mechanism for protein disaggregation by Saccharomyces cerevisiae Hsp104, J. Biol. Chem. 279 (28) (2004) 29139-29146.

[33] T. Haslberger, A. Zdanowicz, I. Brand, J. Kirstein, K. Turgay, A. Mogk, B. Bukau, Protein disaggregation by the AAA + chaperone $\mathrm{ClpB}$ involves partial threading of looped polypeptide segments, Nat. Struct. Mol. Biol. 15 (6) (2008) 641-650.

[34] P. Wendler, J. Shorter, D. Snead, C. Plisson, D.K. Clare, S. Lindquist, H.R. Saibil, Motor mechanism for protein threading through Hsp104, Mol. Cell 34 (1) (2009) 81-92.

[35] E.D. Erben, A. Fadda, S. Lueong, J.D. Hoheisel, C. Clayton, A genome-wide tethering screen reveals novel potential post-transcriptional regulators in Trypanosoma brucei, PLoS Pathog. 10 (6) (2014) e1004178.

[36] S. Lueong, C. Merce, B. Fischer, J.D. Hoheisel, E.D. Erben, Gene expression regulatory networks in Trypanosoma brucei: Insights into the role of the mRNA-binding proteome, Mol. Microbiol. 100 (3) (2016) 457-471.

[37] M. Parsons, E.A. Worthey, P.N. Ward, J.C. Mottram, Comparative analysis of the kinomes of three pathogenic trypanosomatids: Leishmania major, Trypanosoma brucei and Trypanosoma cruzi, BMC Genomics 6 (2005) 127.

[38] M.D. Urbaniak, T. Mathieson, M. Bantscheff, D. Eberhard, R. Grimaldi, D. MirandaSaavedra, P. Wyatt, M.A. Ferguson, J. Frearson, G. Drewes, Chemical proteomic analysis reveals the drugability of the kinome of Trypanosoma brucei, ACS Chem. Biol. 7 (11) (2012) 1858-1865.
[39] S. Kramer, R. Queiroz, L. Ellis, J.D. Hoheisel, C. Clayton, M. Carrington, The RNA helicase DHH1 is central to the correct expression of many developmentally regulated mRNAs in trypanosomes, J. Cell Sci. 123 (Pt 5) (2010) 699-711.

[40] S. Kramer, M. Carrington, An AU-rich instability element in the 3'UTR mediates an increase in mRNA stability in response to expression of a dhh1 ATPase mutant, Translation (Austin) 2 (1) (2014) e28587.

[41] S. Kramer, B. Bannerman-Chukualim, L. Ellis, E.A. Boulden, S. Kelly, M.C. Field, M. Carrington, Differential localization of the two T. brucei poly(A) binding proteins to the nucleus and RNP granules suggests binding to distinct mRNA pools, PLoS One 8 (1) (2013) e54004.

[42] A. Singh, I. Minia, D. Droll, A. Fadda, C. Clayton, E. Erben, Trypanosome MKT1 and the RNA-binding protein ZC3H11: interactions and potential roles in post-transcriptional regulatory networks, Nucleic Acids Res. 42 (7) (2014) 4652-4668.

[43] I. Minia, C. Clayton, Regulating a post-transcriptional regulator: Protein phosphorylation, degradation and translational blockage in control of the trypanosome stress-response RNA-binding protein ZC3H11, PLoS Pathog. 12 (3) (2016) e1005514.

[44] T. An, Y. Liu, S. Gourguechon, C.C. Wang, Z. Li, CDK phosphorylation of translation initiation factors couples protein translation with cell-cycle transition, Cell Rep. 25 (11) (2018) 3204-3214 e5.

[45] M. Fritz, J. Vanselow, N. Sauer, S. Lamer, C. Goos, T.N. Siegel, I. Subota, A. Schlosser, M. Carrington, S. Kramer, Novel insights into RNP granules by employing the trypanosome's microtubule skeleton as a molecular sieve, Nucleic Acids Res. 43 (16) (2015) 8013-8032.

[46] I.D. Tkacz, S.K. Gupta, V. Volkov, M. Romano, T. Haham, P. Tulinski, I. Lebenthal, S. Michaeli, Analysis of spliceosomal proteins in Trypanosomatids reveals novel functions in mRNA processing, J. Biol. Chem. 285 (36) (2010) 27982-27999.

[47] M.D. Urbaniak, Casein kinase 1 isoform 2 is essential for bloodstream form Trypanosoma brucei, Mol. Biochem. Parasitol. 166 (2) (2009) 183-185.

[48] N.G. Jones, E.B. Thomas, E. Brown, N.J. Dickens, T.C. Hammarton, J.C. Mottram, Regulators of Trypanosoma brucei cell cycle progression and differentiation identified using a kinome-wide RNAi screen, PLoS Pathog. 10 (1) (2014) e1003886.

[49] T.C. Hammarton, S. Kramer, L. Tetley, M. Boshart, J.C. Mottram, Trypanosoma brucei Polo-like kinase is essential for basal body duplication, kDNA segregation and cytokinesis, Mol. Microbiol. 65 (5) (2007) 1229-1248.

[50] Y. Wei, Z. Li, Distinct roles of a mitogen-activated protein kinase in cytokinesis between different life cycle forms of Trypanosoma brucei, Eukaryot. Cell 13 (1) (2014) 110-118.

[51] B.M. Mony, P. Macgregor, A. Ivens, F. Rojas, A. Cowton, J. Young, D. Horn, K. Matthews, Genome-wide dissection of the quorum sensing signalling pathway in Trypanosoma brucei, Nature 505 (7485) (2014) 681-685.

[52] S. Michaeli, The response of trypanosomes and other eukaryotes to ER stress and the spliced leader RNA silencing (SLS) pathway in Trypanosoma brucei, Crit. Rev. Biochem. Mol. Biol. 50 (3) (2015) 256-267.

[53] R. Hope, E. Ben-Mayor, N. Friedman, K. Voloshin, D. Biswas, D. Matas, Y. Drori, A. Gunzl, S. Michaeli, Phosphorylation of the TATA-binding protein activates the spliced leader silencing pathway in Trypanosoma brucei, Sci. Signal 7 (341) (2014) ra85.

[54] M.A. Morales, R. Watanabe, M. Dacher, P. Chafey, Y. Osorio, J. Fortea, D.A. Scott, S.M. Beverley, G. Ommen, J. Clos, S. Hem, P. Lenormand, J.C. Rousselle, A. Namane, G.F. Spath, Phosphoproteome dynamics reveal heat-shock protein complexes specific to the Leishmania donovani infectious stage, Proc. Natl. Acad. Sci. U S A 107 (18) (2010) 8381-8386.

[55] P. Tsigankov, P.F. Gherardini, M. Helmer-Citterich, G.F. Spath, D. Zilberstein, Phosphoproteomic analysis of differentiating Leishmania parasites reveals a unique stage-specific phosphorylation motif, J Proteome Res 12 (7) (2013) 3405-3412.

[56] P. Tsigankov, P.F. Gherardini, M. Helmer-Citterich, G.F. Spath, P.J. Myler, D. Zilberstein, Regulation dynamics of Leishmania differentiation: Deconvoluting signals and identifying phosphorylation trends, Mol. Cell Proteomics 13 (7) (2014) 1787-1799. 